Check for updates

Cite this: RSC Adv., 2017, 7, 47049

\title{
A gold nanodendrite-decorated layered double hydroxide as a bifunctional electrocatalyst for hydrogen and oxygen evolution reactions in alkaline media
}

\begin{abstract}
M. Taei, (D) * E. Havakeshian and F. Hasheminasab
In this work, a gold nanodendrites-CuMgFe layered double hydroxide composite (AuNDs(LDH) was introduced as a bifunctional electrocatalyst for hydrogen and oxygen evolution reactions (HER and OER, respectively) in alkaline media. AuNDs@LDH/GCE was prepared by the coating of the surface of a glassy carbon electrode using the CuMgFe-LDH suspension, and followed by Au electrodeposition. Field emission scanning electron microscopy (FE-SEM) images and energy dispersive X-ray spectroscopy (EDX) analysis showed that nanodendrite-like structures of Au are formed on the LDH film, while Au is electrodeposited as nanoparticles on the GCE (AUNPs/GCE). The performance of the catalysts for HER and OER was investigated using polarization curves, Tafel plots and electrochemical impedance spectroscopy (EIS). AuNDs@LDH/GCE exhibited the lowest onset potential and highest current density in comparison with AuNPs/GCE and LDHQAUNPs/GCE for both the OER and HER. The lower values of Tafel slope and charge transfer resistance indicated that the kinetics of charge transfer for the AuNDs@LDH are faster than those for the other studied electrodes. A durability test was performed using chronopotentiometry method that reveals the highest durability of the AuNDsaLDH for both of the reactions. The synergistic effect of the AuNDs and LDH and the high active surface area of AuNDs could be major parameters for enhanced catalytic performance of the AuNDs@LDH. As a result, this strategy provides a promising approach for the fabrication of highly efficient metal nanostructure-decorated LDHs, which can be used as a catalyst in water electrolyzers.
\end{abstract}

Received 18th May 2017

Accepted 2nd October 2017

DOI: $10.1039 / \mathrm{c} 7 \mathrm{ra05625g}$

rsc.li/rsc-advances a critical role in the commercialization of the water electrolyzers. One of the interesting strategies to create such catalysts is the dispersion of costly catalysts onto a non-noble metal support material. This way can minimize the cost of the production of commodity catalysts owing to the decrease of metal dosage. Moreover, the supports and catalytic active components can interact and function through a synergistic effect, which leads to a remarkable enhancement in the catalytic efficiency. $^{4-6}$

Layered double hydroxides (LDHs) are typical layered materials with the general formula of $\left[\mathrm{M}_{(1-x)}{ }^{2+} \mathrm{M}_{x}^{3+}(\mathrm{OH})_{2}\right]^{x+}\left(\mathrm{A}^{n-}\right)_{x / n} \cdot y \mathrm{H}_{2} \mathrm{O}$, where $\mathrm{M}^{2+}$ and $\mathrm{M}^{3+}$ cations occupy octahedral holes in a brucite-like layer and $\mathrm{A}^{n-}$ is located in the hydrated interlayer anion. ${ }^{7}$ LDHs have been proved to be inexpensive, highly active and versatile materials with tunable properties. They have been investigated as catalyst for OER and HER..$^{8-10}$ Moreover, they have the ability to immobilize the metal nanostructures, which makes them good supports in many catalytic reactions. ${ }^{11-13}$ To the best of our knowledge, there are few studies about the investigation of catalytic activity of LDHssupported metal nanostructures in OER and HER, especially; it was found no report for $\mathrm{LDH}$-supported $\mathrm{Au}$ nanostructures. $\mathrm{Au}$ 
nanostructures have high active surface area, excellent electrical conductivity and superior catalytic activity. ${ }^{\mathbf{1 4}}$ Due to these unique characterizations, they (alone or in combination with other materials) have been investigated as good catalysts or electrocatalyst supports for OER ${ }^{15,16}$ and HER. ${ }^{17,18}$

In this work, Au is electrodeposited on CuMgFe-LDH coated on the glassy carbon electrode. Electrodeposition is a direct, simple, quick and inexpensive approach for fabrication of nanostructures on a support. ${ }^{19}$ Then, the surface characterization was performed using field emission scanning electron microscopy (FE-SEM), energy dispersive X-ray spectroscopy (EDX) and cyclic voltammetry (CV). The catalytic activity and durability of the Au nanodendrites-like structures supported on CuMgFe-LDH (AuNDs@CuMgFe-LDH) were studied for both of the OER and HER in alkaline media, and then were compared with those of the electrodeposited $\mathrm{Au}$ and CuMgFe-LDH catalysts using electrochemical methods.

\section{Experimental}

\subsection{Chemicals}

All chemicals were of analytical grade and were purchased from Merck. All solutions were prepared just before use with doubly distilled water.

CuMgFe-LDH was synthesized according to the procedure reported previously. ${ }^{20}$ Briefly, a solution of $0.4 \mathrm{~mol} \mathrm{NaOH}$ in $100 \mathrm{~mL}$ deionized water was added dropwise into a solution containing $\mathrm{Cu}\left(\mathrm{NO}_{3}\right)_{3} \cdot 6 \mathrm{H}_{2} \mathrm{O}, \mathrm{Mg}\left(\mathrm{NO}_{3}\right)_{2} \cdot 6 \mathrm{H}_{2} \mathrm{O}$ and $\mathrm{Fe}\left(\mathrm{NO}_{3}\right)_{3}-$ $.9 \mathrm{H}_{2} \mathrm{O}$ (the molar ratio of $\mathrm{Cu}: \mathrm{Mg}: \mathrm{Fe}$ is $1: 2: 1$ ) in $200 \mathrm{~mL}$ of deionized water with vigorous stirring at $65{ }^{\circ} \mathrm{C}$ until the final $\mathrm{pH}=9$ was obtained. Then, the product was transferred into the stainless steel autoclave for $24 \mathrm{~h}$ at $120^{\circ} \mathrm{C}$. The product was washed with deionized water several times and dried at $80{ }^{\circ} \mathrm{C}$. Finally, the obtained powder was analyzed using X-ray diffractometer (XRD) and an energy dispersive X-ray spectrometer (EDS). The results ${ }^{21}$ were in good agreement with those previously reported, ${ }^{20}$ which showed the successful synthesize of the CuMgFe-LDH. X-ray photoelectron spectroscopy (XPS, Bestec Company, Germany) was used to show the interaction between $\mathrm{LDH}$ and $\mathrm{Au}$ nanostructures.

\subsection{Preparation of modified electrodes}

To obtain a flat surface and remove surface contaminants, the GCE surface was polished with alumina powder slurry $(5 \mu \mathrm{m})$ and then, washed ultrasonically in a mixture of ethanol/distilled water $(1: 1 \mathrm{v} / \mathrm{v})$ for 10 minutes and finally, dried in air. In the next step, $0.10 \mathrm{mg}$ of the synthesized LDH was dispersed ultrasonically in $10 \mathrm{~mL}$ distilled water to obtain a partial suspension. Then, $5 \mu \mathrm{L}$ of the LDH suspension was dropped on the GCE surface and allowed to dry in air at room temperature. Then, Au was electrodeposited on the electrode surface by applying 40 continuous cycles cyclic voltammetry in a potential range of -1.5 to $0 \mathrm{~V}$ with a scan rate of $50 \mathrm{mV} \mathrm{s}^{-1}$ in a solution containing $0.10 \mathrm{~mol} \mathrm{~L}^{-1} \mathrm{KNO}_{3}$ and $4.0 \times 10^{-3} \mathrm{~mol} \mathrm{~L}^{-1}$ $\mathrm{HAuCl}_{4} \cdot 3 \mathrm{H}_{2} \mathrm{O}$. Finally, to increase the adhesion of the coating to the surface, $10 \mu \mathrm{L}$ of Nafion solution $(2.0 \% \mathrm{w} / \mathrm{v})$ was dropped onto the electrode. The prepared electrode was named AuNDs@CuMgFe-LDH/GCE.

The same procedure was used to prepare the other catalysts including AuNPs, CuMgFe-LDH and CuMgFe-LDH@AuNPs on the GCE by changing the electrode preparation sequence. It should be noted that the number of cyclic voltammetry in the step of Au electrodeposition was controlled to load the same Au amounts on all of the above-mentioned electrodes.

\subsection{Surface characterizations}

A field emission scanning electron microscope (FESEM, Hitachi S4160) operated at an accelerating voltage of $25.0 \mathrm{kV}$ equipped with an energy dispersive X-ray spectrometer (EDS) was employed to examine the surface morphology and determine the chemical composition of the prepared electrodes.

\subsection{Electrochemical measurements}

Most electrochemical experiments were performed using a Metrohm instrument, Model 797 VA processor. An Autolab potentiostat-galvanostat, Model PGSTAT302, was used to record chronoamperometric curves and electrochemical impedance spectroscopy (EIS) plots. A conventional three-electrode electrochemical cell consisting of a coated or uncoated GCE with a geometric surface area of $0.0314 \mathrm{~cm}^{2}$ as the working electrode and a platinum wire counter electrode and a reference electrode $(\mathrm{Ag} / \mathrm{AgCl}, \mathrm{KCl} 3.0 \mathrm{M}$ ) was employed to perform all electrochemical measurements. However, all the reported potentials in this work were referenced to the reversible hydrogen electrode (RHE). The geometric surface area of the as-prepared electrodes was used to normalize the current densities. The electrocatalytic activity of the electrode was evaluated in deaerated $1.0 \mathrm{M} \mathrm{KOH}$ solution at room temperature.

\section{Results and discussions}

\subsection{Morphological and surface characterizations}

To compare the surface morphology of the electrodes, FE-SEM images of their surface were taken. As shown in our previous work, ${ }^{21} \mathrm{Au}$ is electrodeposited on the GCE as Au nanoparticles (AuNPs) with an average diameter of $75 \mathrm{~nm}$. AuNPs cover uniformly whole the GCE surface. The CuMgFe-LDH film on the GCE is composed of agglomerates of fine nanoparticles and macropores between them. Fig. 1A shows that the CuMgFe-LDH film casted on the AuNPs/GCE has a similar morphology to the CuMgFe-LDH casted on the GCE. The FE-SEM images from the CuMgFe-LDH/GCE surface after the $\mathrm{Au}$ electrodeposition (Fig. 1B) show that nanodendrites-like structures are formed on the LDH film. Moreover, nanoparticles with the diameter of about $23 \mathrm{~nm}$ are seen at some places. The investigation of EDX spectrums shows that the peaks of $\mathrm{Au}$ appear after the $\mathrm{Au}$ electrodeposition. This indicates the formation of AuNDs on the $\mathrm{LDH} / \mathrm{GCE}$. The peaks of $\mathrm{Cu}, \mathrm{Mg}$ and Fe elements shown in Fig. 1C are also seen before the Au electrodeposition and are attributed to the LDH film. Fig. 1D shows the EDX maps of the SEM image taken from the surface of AuNDs@CuMgFe-LDH/ GCE. Dislike $\mathrm{Mg}$ and $\mathrm{O}, \mathrm{Fe}$ and $\mathrm{Cu}$ are distributed uniformly 

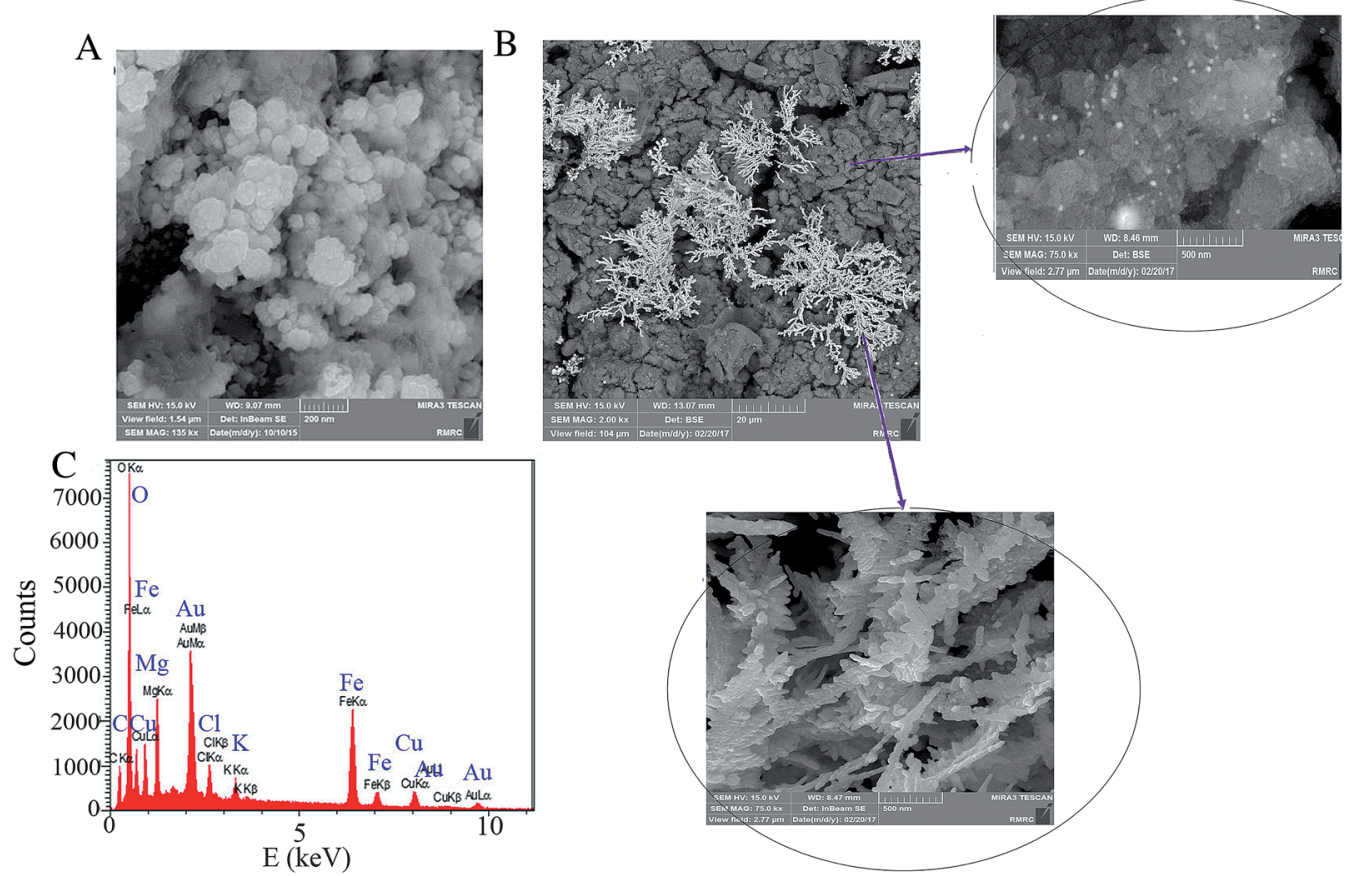

$\mathrm{D}$

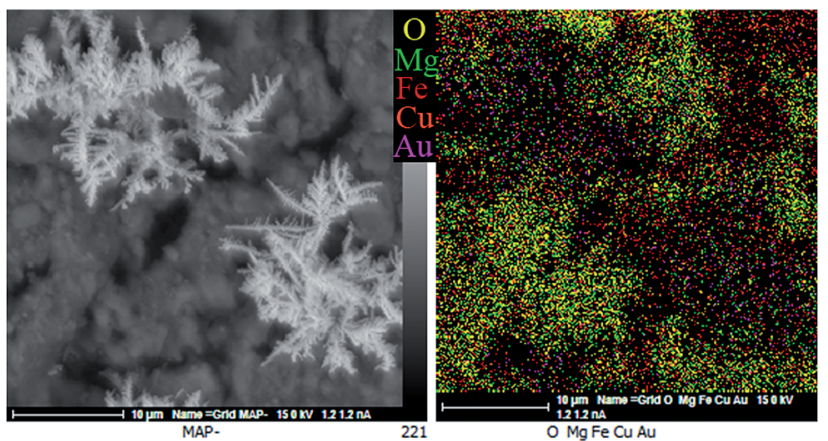

Fig. 1 FE-SEM images of LDH@AuNPs/GCE (A), and AuNDs@LDH/GCE (B); the EDX spectrum (C), and EDX map (D) from AuNDs@LDH/GCE surface.

on the whole of the surface. The dots related to Au have been gathered where there are nanodendrites, and have been distributed in accordance with them, which demonstrates the dendrites-like nanostructures are of $\mathrm{Au}$. The $\mathrm{Au}$ dotes seen in other places, where nanodendrites are not present, can be related to $\mathrm{Au}$ nanoparticles. As it is clearly seen, $\mathrm{Mg}$ covers mainly parts that are lack of $\mathrm{Au}$ nanodendrites. Therefore, it is concluded that AuNDs are formed mainly on LDH sites where a few amount of $\mathrm{Mg}$ is present. Although the used electrodeposition procedure and condition are same for AuNDs@CuMgFe-LDH/GCE and AuNPs/GCE, their morphology are very different. This reveals that the use of CuMgFe-LDH as the substrate induces the formation of AuNDs and reduces the size of AuNPs.

\subsection{Electrochemical characterizations}

The cyclic voltammogram (CV) of the studied electrodes was recorded at scan rate of $50 \mathrm{mV} \mathrm{s}^{-1}$ in the potential range of 0.63 to $2.23 \mathrm{~V}$ (Fig. 2). The CuMgFe-LDH/GCE exhibits a cathodic peak related to the conversion of $\mathrm{Fe}^{2+} / \mathrm{Fe}^{3+}$ associated with $\mathrm{OH}^{-}$at about $1.13 \mathrm{~V}^{22}$ As Au electrodeposited on the $\mathrm{CuMgFe}-\mathrm{LDH} / \mathrm{GCE}$, strong anodic peaks in the potential ranges of 1.10 to $1.60 \mathrm{~V}$ and cathodic peaks in the potential ranges of 0.63 to $1.30 \mathrm{~V}$ appear, which are related to the Au oxidation and $\mathrm{Au}$ oxides/hydroxides reduction reactions, respectively. All of these peaks are also seen for the AuNPs/GCE. However, their

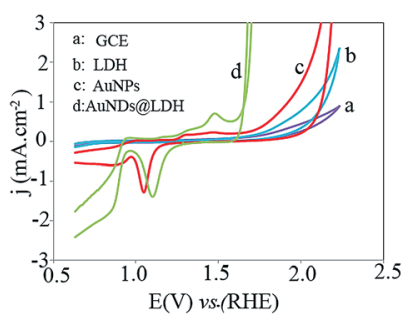

Fig. 2 Cyclic voltammograms of the electrodes in $1.0 \mathrm{M} \mathrm{KOH}$ at the scan rate of $50 \mathrm{mV} \mathrm{s}^{-1}$. 
current density for the AuNDs@LDH is higher than that for the AuNPs catalyst. Moreover, the onset potential of the cathodic peak at the range of 1.0 to $1.3 \mathrm{~V}$ is significantly decreased when $\mathrm{Au}$ is electrodeposited on the CuMgFe-LDH substrate in comparison with AuNPs electrodeposited on the GCE, indicating $\mathrm{Fe}$ hydroxides facilitate the reduction of $\mathrm{Au}$ oxide species. This may be due to the higher electronegativity of $\mathrm{Au}$ with respect to Fe. Therefore, Au oxide species affinity electrons from the CuMgFe-LDH and hence, are reduced at lower potentials. ${ }^{23}$ As it is evident in the figure, the onset potential ( $\left.E_{\text {onset }}\right)$ of oxygen evolution reaction for the AuNPs/GCE is lower than that for the CuMgFe-LDH/GCE. After the electrodeposition of AuNDs on the CuMgFe-LDH/GCE, OER occurs at much lower $E_{\text {onset }}$ than the AuNPs/GCE and CuMgFe-LDH/GCE.

\subsection{Oxygen evolution reaction}

To evaluate the catalytic performance of the electrodes toward OER, the polarization curves of all the prepared electrodes were recorded at a slow scan rate of $5 \mathrm{mV} \mathrm{s}^{-1}$ to minimize the capacitive current, and then corrected for the solution resistance. As shown in Fig. 3A, the AuNDs@LDH/GCE and LDH@AuNPs/GCE exhibit the lower onset potential and the higher current density for OER in comparison with the AuNPs and LDH catalysts. This indicates that there is a synergistic effect between the $\mathrm{Au}$ nanodendrites and the CuFeMg-LDH. For a better comparison, the current densities of the electrodes at a fixed potential of $1.80 \mathrm{~V}$ vs. RHE were summarized in Fig. 3B. As can be seen, the current density is $32 \mathrm{~mA} \mathrm{~cm}{ }^{-2}$ for AuNDs@LDH/GCE, which is 82, 48 and 5 times higher than those for the LDH, AuNPs and LDH@AuNPs catalysts, respectively. Moreover, the potential required to reach $j=10 \mathrm{~mA} \mathrm{~cm}^{-2}$ is $1.76 \mathrm{~V}$ for AuNDs@LDH/GCE, while it is 1.89 and 2.22 for the LDH@AuNPs/GCE and AuNPs/GCE, respectively. Therefore, it indicates that AuNDs-decorated the CuFeMg-LDH have a higher catalytic activity toward OER than the LDH@AuNPs/GCE and the AuNPs deposited on the GCE.

Tafel plots of the electrodes were obtained to study the OER electrochemical kinetics. As shown in Fig. 3C, the Tafel slopes are reduced when both of the Au nanostructures and LDH are inserted on the electrode surface. The AuNDs@LDH/GCE with the Tafel slope of $53 \mathrm{mV} \mathrm{dec}{ }^{-1}$ exhibits much faster electrochemical kinetics toward the OER in comparison with LDH@AuNPs/GCE, AuNPs/GCE and LDH/GCE with slope of 110, 249 and $294 \mathrm{mV} \mathrm{dec}^{-1}$, respectively.

The durability of the electrodes during OER was evaluated using chronopotentiometry test. A constant current density of $3.2 \mathrm{~mA} \mathrm{~cm} \mathrm{~cm}^{-2}$ was applied to the electrode and the plot of potential versus time was recorded. As shown in Fig. 3D, the AuNDs@LDH/GCE exhibits the lowest operating potential. For the AuNPs/GCE, the operating potential increases by some millivolts after 300 min testing, while it is nearly constant within $2000 \mathrm{~s}$ for the AuNDs@LDH and LDH@AuNPs catalysts. It indicates that the synergistic effect of the $\mathrm{Au}$ and $\mathrm{CuMgFe}-$ LDH results in an increase in the durability of the catalysts.

\subsection{Hydrogen evolution reaction}

Fig. 4A depicts the HER polarization curves for all the electrodes at a scan rate of $5 \mathrm{mV} \mathrm{s}^{-1}$. HER doesn't occur significantly at the
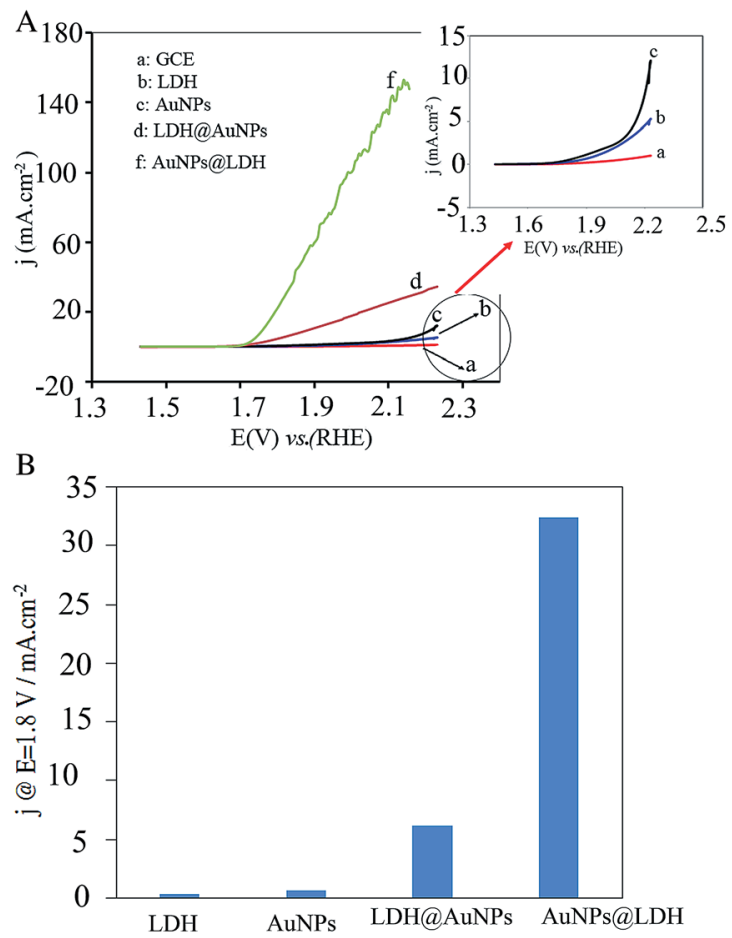
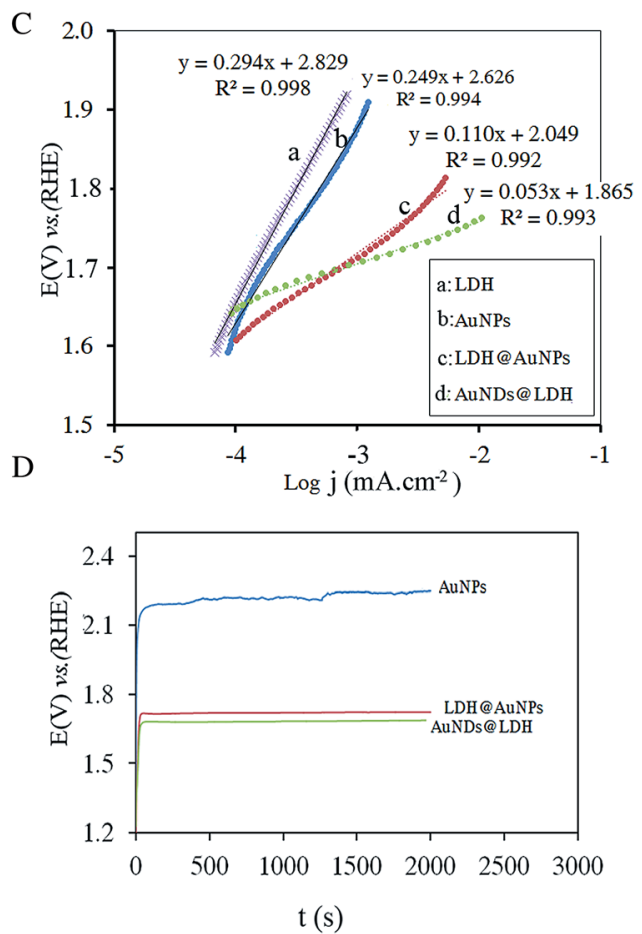

Fig. 3 OER polarization curves of the electrodes at a scan rate of $5 \mathrm{mV} \mathrm{s}^{-1}(\mathrm{~A})$, current densities of the electrodes at a fixed potential of $1.80 \mathrm{~V} v \mathrm{~s}$. RHE (B), OER Tafel plots (C), and chronopotentiometric curves at $0.032 \mathrm{~mA} \mathrm{~cm}^{-2}$ (D), in $1.0 \mathrm{M} \mathrm{KOH}$ solution. 

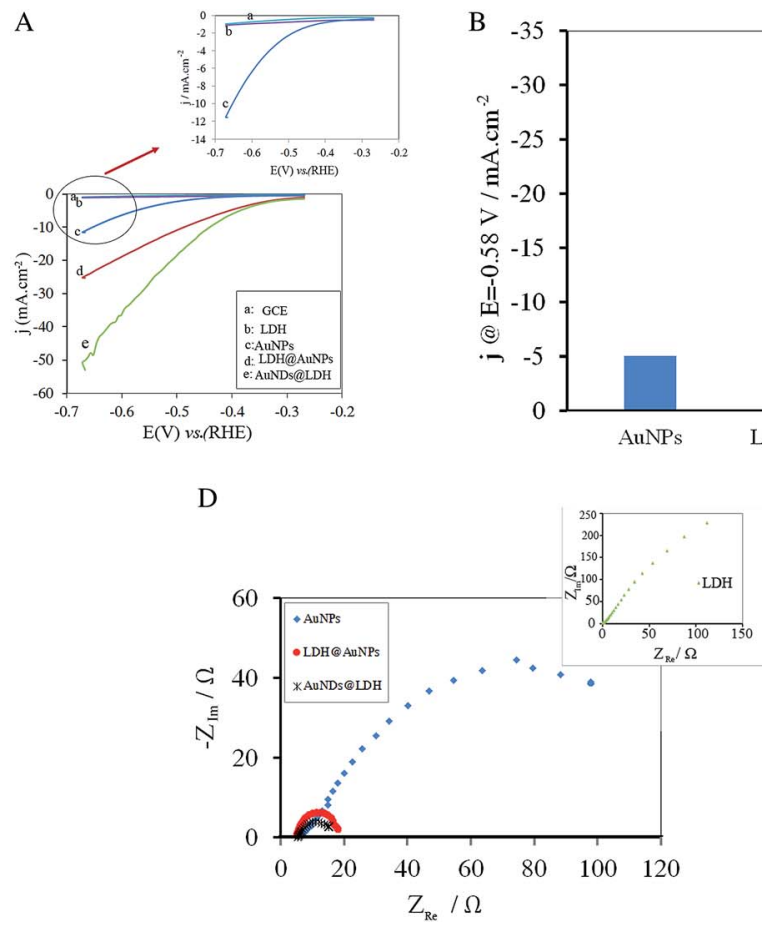
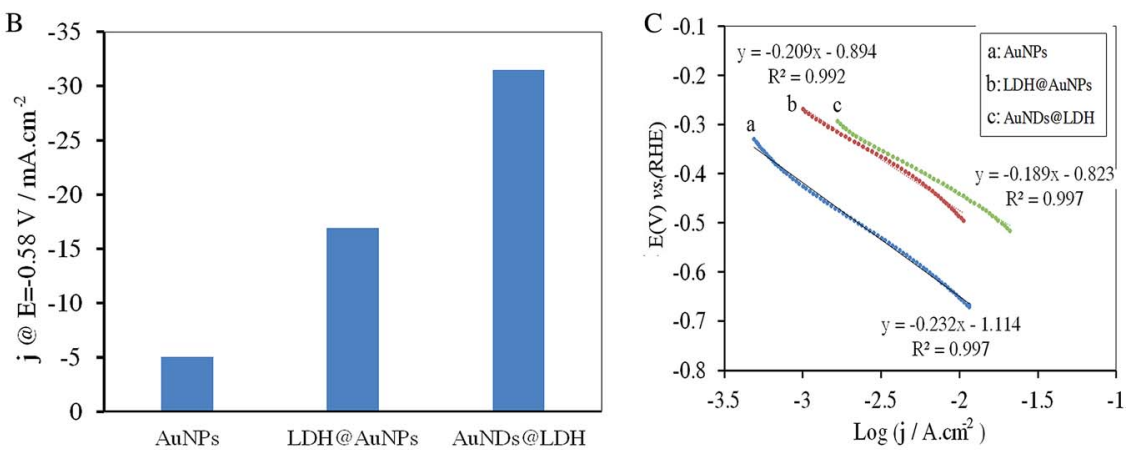

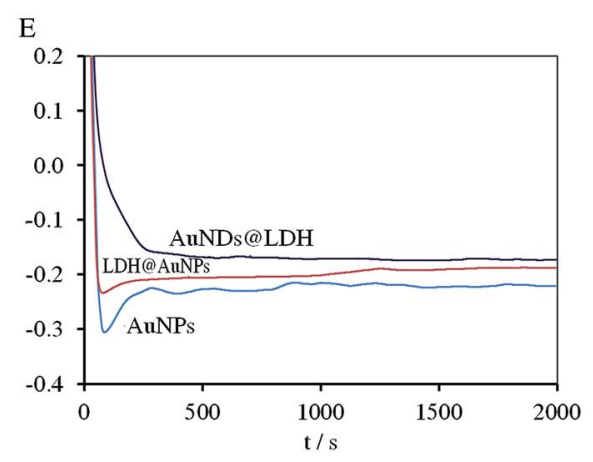

Fig. $4 \mathrm{HER}$ polarization curves of the electrodes at a scan rate of $5 \mathrm{mV} \mathrm{s}^{-1}(\mathrm{~A})$, current densities of the electrodes at a fixed potential of $-0.58 \mathrm{~V}$ vs. RHE (B), HER Tafel plots (C), Nyquist plots at $-1.60 \mathrm{~V}(\mathrm{D})$, and chronopotentiometric curves under a constant current density of $-0.016 \mathrm{~mA}$ $\mathrm{cm}^{-2}(\mathrm{E})$, in $1.0 \mathrm{M} \mathrm{KOH}$ solution.

unmodified GCE and the GCE modified with the CuMgFe-LDH in the potential range from -0.67 to $-0.27 \mathrm{~V}$. Both of the AuNDs@LDH/GCE and LDH@AuNPs/GCE exhibit much lower HER onset potential values and higher current density than that of the AuNPs-modified GCE. The AuNDs@LDH/GCE shows cathodic current density of $31 \mathrm{~mA} \mathrm{~cm} \mathrm{~cm}^{-2}$ at the potential of $-0.58 \mathrm{~V}$, which is about 1.9 and 6.2 times higher than those of LDH@AuNPs/GCE and AuNPs/GCE, respectively (Fig. 4B). The potential at the current density of $10 \mathrm{~mA} \mathrm{~cm}^{-2}$ for AuNPs/GCE, LDH@AuNPs/GCE and AuNDs@LDH/GCE is -0.66, -0.49 and $-0.44 \mathrm{~V}$, respectively, which shows the AuNDs@LDH/GCE exhibits the lowest value. All of the above results indicate that the AuNDs@LDH catalyst has a higher electrocatalytic activity toward HER in comparison with the other studied catalysts.

The comparison of Tafel plots in Fig. 4C reveals that Tafel slope reduces significantly when both of the Au nanostructures and CuMgFe-LDH are present in the catalyst. Moreover, it is found that the AuNDs@LDH has lower Tafel slope than LDH@AuNPs, indicating the higher kinetics of HER at the AuNDs@LDH. The Tafel slope for all the catalysts is close to $120 \mathrm{mV} \mathrm{dec}^{-1}$, indicating that the rate determining step for HER is the proton discharge electrosorption (known as Volmer reaction: $\left.\mathrm{M}+\mathrm{H}_{2} \mathrm{O}+\mathrm{e} \leftrightarrow \mathrm{M}-\mathrm{H}_{\mathrm{ads}}+\mathrm{OH}^{-}\right){ }^{24}$

To further investigate the electrode kinetics under HER process, the EIS measurements were carried out from $10^{5}$ to $10^{-2} \mathrm{~Hz}$ at the AC voltage of $-1.60 \mathrm{~V}$ with amplitude of $10 \mathrm{mV}$. As can be seen in Fig. 4D, all of the catalysts exhibit one semicircle, which shows charge transfer process is the rate determining step (RDS). The diameter of semicircles, which is related to charge transfer resistance, reduces in the order AuNDs@LDH $<$ LDH@AuNPs < AuNPs < CuMgFe-LDH. A lower value corresponds to a faster reaction rate. As a result, HER occurs at the AuNDs@LDH with the highest rate.

The durability of the electrodes during HER was studied using chronopotentiometry method. A constant current density of $-0.016 \mathrm{~mA} \mathrm{~cm}^{-2}$ was applied to the electrode for $2000 \mathrm{~s}$. As can be seen in Fig. 4E, in comparison with the other catalysts, AuNDs@LDH exhibits lower potential with negligible oscillation and hence, the higher durability.

\subsection{Effective parameters}

As found from the obtained results in the previous sections, the LDH@AuNPs and AuNDs@LDH exhibit higher catalytic performance for OER and HER as compared with the AuNPs and CuFeMg-LDH. It is well known that LDHs are potential candidates to catalysis OER and HER. ${ }^{9,25,26}$ However, they suffer from their inherent low electrical conductivity. Despite LDHs, $\mathrm{Au}$ has good electrical conductivity. Moreover, it is accounted as an appropriate catalyst that has strong affinity to oxygen and/or oxygen-containing groups $\left(\right.$ e.g. $\left.\mathrm{OH}_{\mathrm{ad}}{ }^{-}\right) \cdot{ }^{27,28}$ Therefore, the $\mathrm{Au}$ nanostructures at the LDH@AuNPs and AuNDs@LDH, in addition to the increase of electrical conductivity, can catalysis OER and HER. Moreover, since Au is the most electronegative metal, it affinities strongly the electrons of CuMgFe-LDH and thus facilitates the oxidation of the metal oxides. This leads to a promotion in the formation of transition-metal ions at higher oxidation states, which are accounted as active sites for OER and HER. ${ }^{16,23}$ 


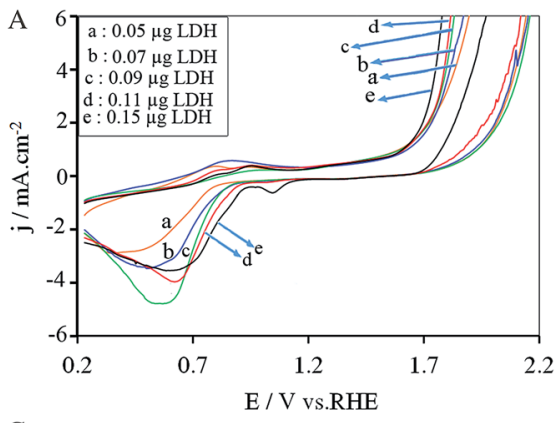

C

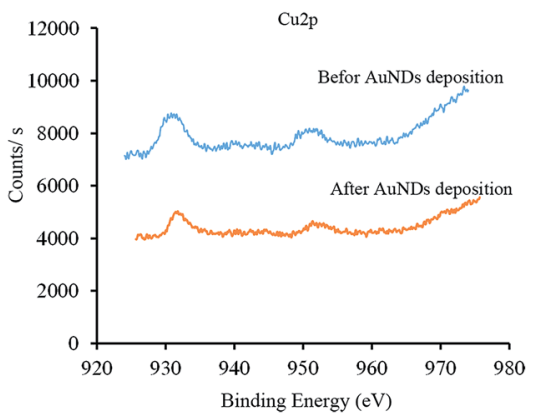

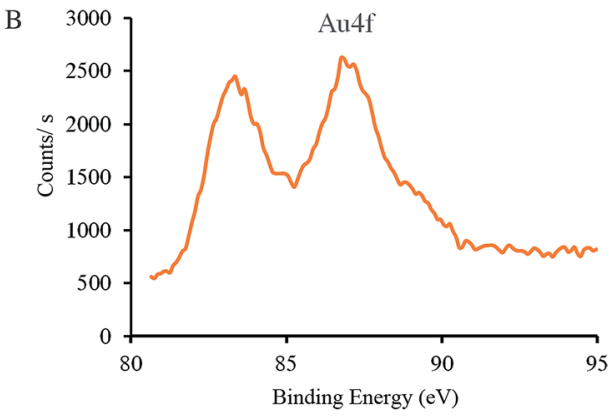

D

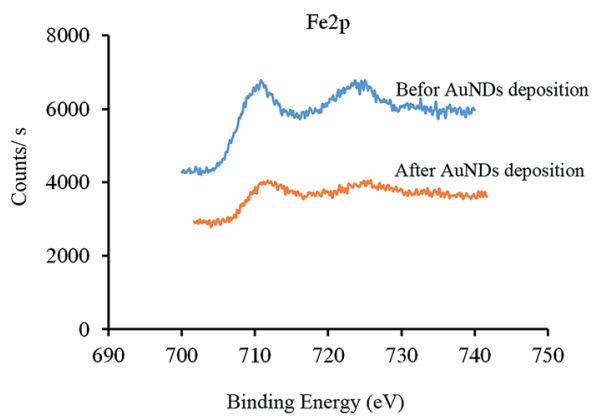

Fig. 5 Cyclic voltammograms of the AuNDs@LDH/GCE with different amounts of LDH in $1.0 \mathrm{M} \mathrm{KOH}$ at scan rate of $50 \mathrm{mV} \mathrm{s}{ }^{-1}$ (A); and XPS spectrums for $\mathrm{Au}(\mathrm{B}), \mathrm{Cu}(\mathrm{C})$, and Fe (D).

The electronic interactions within the CuFeMg-LDH and $\mathrm{Au}$ nanostructures can be observed experimentally using cyclic voltammetry method. Some electrodes with different amounts of LDH and constant amount of Au were fabricated. Cyclic voltammogram of each electrode was obtained (Fig. 5A). It is observed that the cathodic peaks related to the $\mathrm{Au}$ oxides/ hydroxides reduction reactions in the range of 0.20 to $1.0 \mathrm{~V}$ are shifted to more positive potentials as LDH loading is increasing. Moreover, the overpotential of OER decreases by increase in LDH amount. All of these results suggest an electronic interaction between the $\mathrm{LDH}$ and $\mathrm{Au}$ nanostructures. ${ }^{15,29}$ Moreover, X-ray photoelectron spectroscopy (XPS) was employed to determine the valence state and interaction of metals/metal ions. Fig. 5B shows the XPS spectrums of $\mathrm{Au} 4 \mathrm{f}$ before and after the Au electrodeposition on the LDH/GCE. After electrodeposition, two main peaks are observed at 83.3 and $87.0 \mathrm{eV}$, corresponding to the binding energies of $\mathrm{Au}$ $4 f_{7 / 2}$ and $A u 4 f_{5 / 2}$, respectively. The XPS characteristic peaks for metallic $\mathrm{Au}^{0}$ appear at 84.0 and $87.7 \mathrm{eV}^{30}$ Therefore, this suggests that the $\mathrm{Au}$ nanostructures are electrodeposited as $\mathrm{Au}^{0}$ on the LDH/GCE. The negative shift $(0.7 \mathrm{eV})$ indicates that the electron transfer is done from the LDH to the AuNSs. XPS spectrums of $\mathrm{Cu} 2 \mathrm{p}$ and $\mathrm{Fe} 2 \mathrm{p}$ before and after the Au electrodeposition are also compared in Fig. 5C and D, respectively. Binding energies at $930.7\left(\mathrm{Cu} 2 \mathrm{p}_{3 / 2}\right)$ and $951.6 \mathrm{eV}\left(\mathrm{Cu} 2 \mathrm{p}_{1 / 2}\right)$ are related to $\mathrm{Cu}(\mathrm{OH})_{2}$ species. Two main peaks at $711.7\left(\mathrm{Fe} 2 \mathrm{p}_{3 / 2}\right)$ and $724.1 \mathrm{eV}\left(\mathrm{Fe} 2 \mathrm{p}_{1 / 2}\right)$, indicating the presence of the $\mathrm{Fe}^{3+}$ cation. ${ }^{31}$ After Au electrodeposition, these peaks shift to the higher energies, demonstrating the electron transfer from the LDH to AuNSs. All of these results confirm the presence of an electronic interaction between the LDH and AuNSs, which induces the synergistic effects of the Au nanostructures and CuFeMg-LDH in OER and HER. Therefore, it results in a decrease in $E_{\text {onset }}$ and an increase in the current density and hence, an enhancement in catalytic activity and durability of the catalysts.

To find why the catalyst performance of AuNDs@LDH is better than LDH@AuNPs, the electrochemically active surface area provided using AuNDs and AuNPs $\left(S_{a}\right)$ for each electrode was calculated using $S_{a}\left(\mathrm{~cm}^{2}\right)=Q / 390$, where the value of $390 \mu \mathrm{C}$ $\mathrm{cm}^{2}$ refer to the charge required to reduce AuO monolayer on the unit surface area of the electrode and $Q$ is the total charge corresponding to the reduction of AuO monolayer formed on the electrode surface. ${ }^{32}$ The value of $Q$ was obtained by recording cyclic voltammetry of each electrode in $0.10 \mathrm{M} \mathrm{H}_{2} \mathrm{SO}_{4}$ and then, the estimation of the area of the peak related to the reduction of Au oxide species. The value of $S_{a}$ is obtained to be $0.029,0.025$ and $0.052 \mathrm{~cm}^{2}$ for the AuNPs, LDH@AuNPs and AuNDs@LDH, respectively. Due to the coating of the AuNPs active sites via the LDH, $S_{a}$ for the LDH@AuNPs is lower than that of the AuNPs. $S_{a}$ for AuNDs@LDH is much higher than that of AuNPs, due to the nanodendrite-like structure of Au electrodeposited on the LDH. Indeed, the nanodendrites provide higher accessible active sites for the reactions, which results in higher current density for the AuNDs@LDH catalyst with respect to the LDH@AuNPs.

\section{Conclusion}

Gold nanodendrites were fabricated simply on CuMgFe-LDH film-coated GCE (AuNDs@LDH/GCE) through electrodeposition method and then, its catalytic activity for OER and HER was 
investigated in $1.0 \mathrm{M} \mathrm{KOH}$. The results showed that the $\mathrm{LDH}$ substrate plays a critical role to electrodeposit $\mathrm{Au}$ as nanodendrites. The AuNDs@LDH catalyst has higher catalytic performance and durability when compared with AuNPs/GCE and LDH@AuNPs/GCE. This enhancement in catalytic activity could be related to synergistic effects between the LDH and AuNDs and high active surface area provided by AuNDs. Accordingly, the AuNDs@LDH can be used as a bifunctional electrocatalyst for the overall water-splitting. This strategy could be extended for the preparation of other metal-coupled $\mathrm{LDH}$ materials for a broad range of technological applications.

\section{Conflicts of interest}

There are no conflicts to declare.

\section{References}

1 S. H. Hong, S. H. Ahn, I. Choi, S. G. Pyo, H. J. Kim, J. H. Jang and S. K. Kim, Appl. Surf. Sci., 2014, 307, 146-152.

2 B. S. Yeo and A. T. Bel, J. Phys. Chem. C, 2012, 116, 8394-8400.

3 Y. Fang, X. Li, Y. Hu, F. Li, X. Lin, M. Tian, X. An, Y. Fu, J. Jin and J. Ma, J. Power Sources, 2015, 300, 285-293.

4 C. H. Zhou, Appl. Clay Sci., 2011, 53, 87-96.

5 B. Zhang, J. Fang, J. Li, J. J. Lau, D. Mattia, Z. Zhong, J. Xie and N. Yan, Chem.-Asian J., 2016, 11, 532-539.

6 J. Fang, J. Li, B. Zhang, X. Yuan, H. Asakura, T. Tanaka, K. Teramura, J. Xie and N. Yan, Nanoscale, 2015, 7, 63256333.

7 Y. Kuang, L. Zhao, S. Zhang, F. Zhang, M. Dong and S. Xu, Materials, 2010, 3, 5220-5235.

8 Z. Liu, C. Yu, X. Han, J. Yang, C. Zhao, H. Huang and J. Qiu, ChemElectroChem, 2016, 3, 906-912.

9 W. Ma, R. Ma, C. Wang, J. Liang, X. Liu, K. Zhou and T. Sasaki, ACS Nano, 2015, 9, 1977-1984.

10 L. Qian, Z. Lu, T. Xu, X. Wu, Y. Tian, Y. Li, Z. Huo, X. Sun and X. Duan, Adv. Energy Mater., 2015, 5, 1500245.

11 J. Zhao, M. Shao, D. Yan, S. Zhang, Z. Lu, Z. Li, X. Cao, B. Wang, M. Wei, D. G. Evans and X. Duan, J. Mater. Chem. A, 2013, 1, 5840-5846.

12 L. Sobhana, M. Sarakha, V. Prevot and P. Fardim, Appl. Clay Sci., 2016, 134, 120-127.

13 A. Mignani, B. Ballarin, M. Giorgetti, E. Scavetta, D. Tonelli, E. Boanini, V. Prevot, C. Mousty and A. Iadecola, J. Phys. Chem. C, 2013, 117, 16221-16230.
14 J. Fang, B. Zhang, Q. Yao, Y. Yang, J. Xie and N. Yan, Coord. Chem. Rev., 2016, 322, 1-29.

$15 \mathrm{~J} . \quad$ W. D. Ng, M. García-Melchor, M. Bajdich, P. Chakthranont, C. Kirk, A. Vojvodic and T. F. Jaramillo, Nat. Energy, 2016, 1, 16053-16061.

16 Y. Zhou and H. C. Zeng, J. Phys. Chem. C, 2016, 120, 2934829357.

17 J. Lu, W. Zhou, L. Wang, J. Jia, Y. Ke, L. Yang, K. Zhou, X. Liu, Z. Tang, L. Li and S. Chen, ACS Catal., 2016, 6, 1045-1053.

18 T. Brülle, W. Ju, P. Niedermayr, A. Denisenko, O. Paschos, O. Schneider and U. Stimming, Molecules, 2011, 16, 1005910077.

19 Nanostructured materials in electrochemistry, ed. A. Eftekhari, John Wiley \& Sons, 2008.

20 S. Xia, L. Zhang, X. Zhou, G. Pan and Z. Ni, Appl. Clay Sci., 2015, 114, 577-585.

21 M. Taei, E. Havakeshian, F. Abedi and M. Movahedi, Int. J. Hydrogen Energy, 2016, 41, 13575-13582.

22 M. Shao, F. Ning, J. Zhao, M. Wei, D. G. Evans and X. Duan, Adv. Funct. Mater., 2013, 23, 3513-3518.

23 Z. Zhuang, W. Sheng and Y. Yan, Adv. Mater., 2014, 26, 39503955.

24 G. Kreysa and B. Hakansson, J. Electroanal. Chem., 1986, 201, 61-83.

25 M. Gong, Y. Li, H. Wang, Y. Liang, J. Z. Wu, J. Zhou, J. Wang, T. Regier, F. Wei and H. Dai, J. Am. Chem. Soc., 2013, 135, 8452-8455.

26 X. Long, J. Li, S. Xiao, K. Yan, Z. Wang, H. Chen and S. A. Yang, Angew. Chem., 2014, 126, 7714-7718.

27 F.-Q. Shao, X.-X. Lin, J.-J. Feng, J. Yuan, J.-R. Chen and A.-J. Wang, Electrochim. Acta, 2016, 219, 321-329.

28 A. Morozan, V. Goellner, A. Zitolo, E. Fonda, B. Donnadieu, D. Jones and F. Jaouen, Phys. Chem. Chem. Phys., 2015, 17, 4047-4053.

29 M. W. Louie and A. T. Bell, J. Am. Chem. Soc., 2013, 135, 12329-12337.

30 S. K. Movahed, M. Fakharian, M. Dabiri and A. Bazgir, RSC Adv., 2014, 4, 5243-5247.

31 W. Gao, Y. Zhao, J. Liu, Q. Huang, S. He, C. Li, J. Zhao and M. Wei, Catal. Sci. Technol., 2013, 3, 1324-1332.

32 S. Trasatti and O. A. Petrii, J. Electroanal. Chem., 1992, 327, 353-376. 